\title{
Mehr Gleichstellung - auch ohne mehr Profit
}

\section{Prof. Dr. Friederike Maier}

Direktorin, Harriet Taylor Mill-Institut, Hochschule für Wirtschaft und Recht, Berlin

Sehr geehrte Frau Pisal, sehr geehrte Damen und Herren,

ich möchte mich für die Einladung zu dieser Veranstaltung ganz herzlich bedanken und freue mich, Ihnen hier als Vertreterin der Wissenschaft meine Position erläutern zu dürfen.

Wir am Harriet Taylor Mill-Institut der Hochschule für Wirtschaft und Recht beschäftigen uns schon lange mit dem Zusammenhang zwischen ökonomischen Fragen und den Geschlechterverhältnissen, so wie es auch unsere Namensgeberin Mitte des neunzehnten Jahrhunderts getan hat. Und auch bei ihren Arbeiten, die weitgehend nur unter dem Namen ihres Mannes John Stuart Mill bekannt sind, ging es um den Zugang der Frauen zu allen Ämtern und beruflichen Positionen, ein Thema, das uns heute ja immer noch beschäftigt.

Zunächst einmal vorweg: Die Daten und Fakten zur Lage in deutschen Unternehmen sind eindeutig und sie haben sich über die letzen 20 Jahre kaum verändert. Wir haben es in den Führungspositionen und insbesondere in den Aufsichtsräten und Vorständen der deutschen Wirtschaft mit einen hochgradig segregierten Arbeitsmarktsegment zu tun - hochgradig segregiert bedeutet, dass der Anteil der Menschen, die in der Minderheit sind, weniger als 20 Prozent beträgt. Nicht nur die Führungspositionen in der Wirtschaft, sondern auch die in den Wissenschaften, den Medien oder in der Justiz sind Männerdomänen. Es gibt auf allen Arbeitsmärkten Segregation: Der Markt für Pflegekräfte ist hochgradig segregiert, der Markt für Automechaniker auch. Es gibt jedoch auch gemischte Bereiche, zum Beispiel die Ärzteschaft oder den Banken- und Versicherungssektor. Veränderungen in der Zusammensetzung der Berufe ereignen sich manchmal schnell wie bei den kaufmännischen Berufen, manchmal langsam und über einen längeren Zeitraum wie in der Ärzteschaft. Das Phänomen, über das wir heute sprechen, nennen wir vertikale Segregation, das heißt die ungleiche Besetzung von Führungspositionen bis hin zu Vorständen und Aufsichtsräten. Dieses Phänomen ist extrem stabil, über alle Branchen hinweg, in privaten wie öffentlichen Unternehmen, in Frauenbranchen, gemischten und Männerbranchen und Berufen, in DAX-Unternehmen und mittelständischen Familienunternehmen, in Banken und Versicherungen et cetera: Der Frauenanteil liegt nirgendwo über 20 Prozent, alles hochgradig männerdominierte Positionen und alles Positionen mit erheblicher ökonomischer und politischer Macht, Einfluss und Prestige.

Für mich als Ökonomin hat dieses Phänomen immer zwei Seiten: Warum ist dieses so, obwohl wir es auf der Angebotsseite, das heißt bei den Frauen und Männern, mit erheblichen Veränderungen zu tun haben? Die Bildungsreformen der 1960erJahre waren vor allem an diesem Punkt sehr erfolgreich, es gibt in vielen akademischen Berufen heute ausreichend qualifizierte Frauen. Auch das norwegische Beispiel hat gezeigt, dass die neuen Aufsichtsrätinnen höher qualifiziert sind und nicht nur jünger und weniger erfahren als ihre männlichen Vorgänger. Es gibt keinen Grund zu der Annahme, dass es unter diesen Frauen weniger kluge, ambitionierte und an einer Führungsposition interessierte gibt als unter den Männern. Auch von den Männern wird ja nicht jeder Führungskraft oder gar Vorstand oder Aufsichtsrat. Aus soziologischen Untersuchungen wissen wir, dass der Zugang zu diesen Positionen auch für Männer hochgradig selektiv ist und viel zum Beispiel mit familiärem Hintergrund, der Zugehörigkeit zu den richtigen Netzwerken zu tun hat. Eher selten sind auch bei Männern „Karrieren außer der Reihe“ oder Seiteneinstiege. Und dennoch gelingt es den Frauen, die mit den richtigen gleichen Merkmalen „ausgestattet“ sind, nicht, in diese Positionen zu kommen. Das hängt eindeutig mit der Nachfrageseite zusammen, das heißt den Unternehmen, den handelnden Akteuren in den Unternehmen, die daran entweder nicht interessiert sind oder aber nicht in der Lage, diesen $\mathrm{Zu}$ stand zu ändern.

Die Männerklubs funktionieren nach Normen und Werten, die wir als soziale Kategorisierung beschreiben: Männlich dominierte Gruppen produzieren Zugangs- und Zugehörigkeitsregeln, die Frauen, entweder weil sie abweichendes Verhalten an den Tag legen oder es ihnen zumindest unterstellt wird, zu Außenseiterinnen machen - die Wahrnehmung als die Fremden und Anderen wiederum kann zu Reibungsverlusten, zu Kommunikationsproblemen, zu Problemen der Kooperation führen. Verbunden mit der Beobachtung, dass Gruppen oft zur Rekrutierung der Gleichen führen und Frauen in diesen homogenen Gruppen als besonders randständig einerseits und sichtbar andererseits wahrgenommen werden, wenn sie nur als Einzelne vertreten sind, führt dazu, dass auftretende Probleme oft einseitig den Frauen zugeschrieben werden. Die Sichtbarkeit einzelner Frauen ist extrem hoch und ihr Handeln wird immer als das Handeln der gesamten Gruppe bewertet. Wie viele Männer sind als Vorstandsvorsitzende schon sang- und klanglos gescheitert, ohne dass die Führungskompetenz von Männern im allgemeinen in Frage gestellt wurde, aber wie viel Aufmerksamkeit und Häme haben die wenigen Frauen erfahren, denen es ähnlich ging?

Das eben beschriebene Phänomen führt mich zu einer weiteren Bemerkung: Unter den oben beschriebenen Umständen ist es nicht verwunderlich, dass die wenigen Studien, die es zu den ökonomischen Folgen gemischter Aufsichtsräte und/ oder Vorstände gibt, wenig eindeutige Ergebnisse haben. Es ist wenig plausibel, warum die wenigen Frauen ökonomisch nun erfolgreicher agieren sollten als die reinen Männerklubs. Um den Einfluss der gemischten Boards auf die ökonomische Entwicklung eines Unternehmens seriös überprüfen zu können, bedarf es zweierlei: Erst einmal tatsächlich gemischte Boards und nicht nur einzelne Frauen in den Aufsichtsgremien/Vorständen 
weniger Unternehmen, und zweitens klare Analysen, welche Faktoren die ökonomische Entwicklung eines Unternehmen beeinflussen. Hier wird man dann feststellen, dass schon die Definition dessen, was ökonomischer Erfolg ist, nicht ganz einfach ist, und dass zudem so viele Faktoren darauf einwirken, ob zum Beispiel die Aktienkurse steigen oder fallen, dass es methodisch sehr ausgefeilter Analysen bedarf, um den Einfluss des Boards $\mathrm{zu}$ isolieren von all den anderen: Turbulenzen an den Börsen, generelle oder branchenspezifische Einbrüche in der Nachfrage, Fehler im Investitionsverhalten et cetera. Hier werden die Entwicklungen in Norwegen als dem Land mit der bisher radikalsten Veränderung sicherlich genau zu untersuchen sein und könnten in einigen Jahren tatsächlich Anhaltspunkte bieten für fundierte Analysen.

Es ist aus Sicht der Wissenschaft also schwierig, beim heutigen Zustand zu sagen: Mehr Frauen in Führungspositionen, in Vorständen und Aufsichtsräten führen zu besseren Renditen, mehr Gewinnen, einem besseren Arbeitsklima, et cetera. Die Forderung nach Gleichstellung, nach Zugang zu allen Positionen in Wirtschaft und Gesellschaft unabhängig vom Geschlecht ist aus meiner Sicht aber auch dann berechtigt, wenn mehr Frauen nicht unbedingt zu mehr Profit beitragen - oder will jemand ernsthaft behaupten, dass Gleichstellung nur dann zu fordern ist wenn es als „business case“ wirkt? Im Gegenteil: Aus meinen Erkenntnissen über das Funktionieren von Gleichstellung folgen folgende Empfehlungen, wie der Wandel gelingen kann:

1. Es geht um den gleichberechtigten Zugang von Frauen zu allen Positionen in Wirtschaft und Gesellschaft;

2. Die Unternehmen nicht nur in Deutschland sind von sich aus nur begrenzt in der Lage, diese Anforderung erfüllen zu können;

3. Deswegen bedarf es verbindlicher Regeln, die durch das Parlament beschlossen werden müssen, denn es handelt sich um die Realisierung elementarer Prinzipien unserer Demokratie;

4. diese verbindlichen Regeln müssen so definiert sein, dass keine kleinen Minderheiten entstehen, es muss gleich eine kritische Masse erreicht werden;

5. Das heißt ich spreche mich dafür aus, verbindliche Quoten festzulegen, die in einem definierten Zeitraum erreicht wer- den müssen; so, wie das einige europäische Länder, nicht nur Norwegen, schon beschlossen haben.

6. Die ökonomische Entwicklung der bundesdeutschen Unternehmen kann davon positiv beeinflusst werden; ob das passiert, das werden wir dann sehen. Ein unternehmerisches Scheitern wegen zu vieler Frauen „an Bord“ ist dagegen noch nicht berichtet worden. Auch der letzte große Insolvenzfall Schlecker hat mit den vielen Frauen in der Belegschaft jahrzehntelang erhebliche Gewinne gemacht - die Fehlentscheidungen haben nicht die Frauen zu verantworten, die Folgen dieser Fehlentscheidungen allerdings schon!

7. Unabhängig von einer gesetzlichen Quotenregelung wird es unumgänglich sein, die personalpolitischen Strategien in den Unternehmen zu verändern, denn nicht nur an der Spitze der Unternehmen steht Wandel an, sondern auch im Personalmanagement insgesamt: Eine größere Vielfalt in den Belegschaften und im Führungspersonal muss überlegt „gemanagt“ werden, denn Vielfalt hat viele positive Seiten, diese kommen aber nicht automatisch zum Tragen, sondern müssen aktiv befördert werden.

Deswegen ist mein Plädoyer, gemeinsam mit vielen anderen Wissenschaftler/inne/n:

- Gleichstellung und Vielfalt müssen in den Unternehmenszielen und Leitbildern verankert sein;

- Es müssen verbindliche Zeitpläne mit Verantwortlichkeiten und Anreizen beziehungsweise Sanktionen festgelegt werden;

- Die Transparenz bei der Besetzung von Führungspositionen, insbesondere in Top-Positionen, muss erhöht werden;

- Die Personalentwicklung muss so gestaltet werden, dass Frauen in die Karriereförderung einbezogen werden, flexiblere Karrieremöglichkeiten für Männer wie Frauen entwickelt werden und Familienarbeit für beide Geschlechter als ein positiver Faktor in der Personalentwicklung verankert wird;

- Man muss die herrschenden Geschlechterstereotype in den Unternehmenskulturen aktiv hinterfragen und damit offener und moderner auf die Lebenswirklichkeit der Menschen eingehen.

Nichts spricht dagegen, dass dies den Unternehmen, gleich welcher Branche, nutzen wird und alles spricht dafür, dass dies ein Beitrag zu mehr Geschlechtergerechtigkeit sein wird.

\section{Wie kann die angemessene Teilhabe erreicht werden?}

\author{
Monika Schulz-Strelow \\ Präsidentin von FidAR - Frauen in die Aufsichtsräte e.V., Berlin \\ Sehr geehrte Frau Pisal, \\ sehr geehrter Herr Botschafter, \\ diese Frage beschäftigt uns gemeinsam seit Jahren. Herz- \\ lichen Dank, dass wir hier im März, dem Monat der vielen
}

politischen Proklamationen am Internationalen Frauentag und notwendigen Aktionen zum Equal Pay Day, aber wenig sichtbaren Veränderungen, zusammenkommen und uns aus unterschiedlicher Sicht mit der aktuellen Situation von Frauen in Aufsichtsräten oder besser von fehlenden Frauen befassen.

Herr Botschafter, wir erinnern uns an das 1. FidAR-Forum im September 2009 in der britischen Botschaft, wo wir leicht 